\title{
Blockchain e a Perspectiva Tecnológica para a Administração Pública: Uma Revisão Sistemática
}

\author{
Blockchain and a Technological Perspective for Public Administration: \\ A Systematic Review
}
Luzia Menegotto Frick de Moura ${ }^{1 \odot}$ Daniela Francisco Brauner ${ }^{1} \odot$ Raquel Janissek-Muniz ${ }^{2}$

\section{RESUMO}

Objetivo: a revisão sistemática desenvolvida neste artigo teve como objetivo apresentar as potenciais aplicações e consequências do uso da Blockchain para a administração pública. Método: a partir da busca de artigos nas bases Scopus, Web of Science, SSRN e Science Direct, e seguindo o método PRISMA, foram selecionados 16 artigos que continham as temáticas: Blockchain, participação governamental direta ou como principal regulamentador, política pública. Resultados: as principais aplicações encontradas foram referentes a: processamento de dados e segurança de dados públicos, novas propostas de regulamentação estatal e de organização institucional. Quanto aos impactos, destacam-se a melhoria na gestão dos dados, diminuição da burocracia e necessidade de afinar a relação entre Estado, sociedade e mercado. Conclusão: a Blockchain apresenta-se como uma tecnologia capaz de renovar os processos de gestão, mas criando novos desafios à administração pública.

Palavras-chave: blockchain; administração pública; governo eletrônico. Classificação JEL: O14, H83, L86.

' Universidade Federal do Rio Grande do Sul, Escola de Administração, Porto Alegre, RS, Brasil.

${ }^{2}$ Universidade Federal do Rio Grande do Sul, Escola de Administração, Programa de Pós-graduação em Administração, Porto Alegre, RS, Brasil.

\section{ABSTRACT}

Objective: the systematic review of this article aimed to present the applications and consequences of using Blockchain for public administration. Method: from researching articles in the Scopus, Web of Science, SSRN and Science Direct databases, and following the PRISMA method, 16 articles were selected that contain the following themes: Blockchain, direct government participation or as the main regulator, public policy. Results: the main applications used were related to: data processing and public data security, required new applications and institutional organization. As for the impacts, we highlight the improvement in data management, reduction of bureaucracy and the need for a relationship between the State, society, and market. Conclusion: blockchain is a technology capable of renewing management processes, creating new challenges for public administration.

Keywords: blockchain; public administration; e-government.

Como citar: Moura, L. M. F. de, Brauner, D. F., \& Janissek-Muniz, R. (2020). Blockchain and a technological perspective for public administration: A systematic review. Revista de Administração Contemporânea, 24(3), 259-274. https://doi.org/10.1590/1982-7849rac2020190171

Editor-chefe: Wesley Mendes-Da-Silva (Fundação Getulio Vargas, EAESP, Brasil) Pareceristas: Fernanda da Silva Momo (Universidade Federal do Rio Grande do Sul, FCE, Brasil) Amanda Cainelli (Universidade Federal do Rio Grande do Sul, Brasil) Natália Marroni Borges (Universidade Federal do Rio Grande do Sul, Brasil) Aceite em: 02/11/2019

\# de revisores convidados até a decisão:

\begin{tabular}{|c|c|c|c|c|c|c|c|c|c|}
\hline & 1 & 2 & 3 & 4 & 5 & 6 & 7 & 8 & 9 \\
\hline $1^{a}$ rodada & $\stackrel{\theta}{2}$ & $\stackrel{\theta}{\theta}$ & $\stackrel{9}{2}$ & & & & & & \\
\hline $2^{a}$ rodada & (x) & 电 & & & & & & & \\
\hline $3^{a}$ rodada & $\stackrel{9}{6}$ & & & & & & & & \\
\hline $4^{a}$ rodada & 9 & & & & & & & & \\
\hline
\end{tabular}




\section{INTRODUÇÃO}

Há décadas que a tecnologia vem impactando a maneira como o governo e a sociedade se relacionam. Seguindo os avanços tecnológicos, novas ferramentas de gestão são incorporadas às organizações públicas ou privadas. No governo federal, por exemplo, o Governo Eletrônico introduziu as tecnologias da informação para otimizar e agilizar os serviços prestados e melhor se comunicar internamente (órgãos) e externamente (sociedade) (Chahin, 2004), viabilizando o acesso à informação e a melhoria na transparência governamental. Melati e Janissek-Muniz (2019), em estudo sobre dimensões do Governo Inteligente, tratam da importância do reconhecimento e aplicação da tecnologia para organização de dados e informações públicas, sugerindo sua relevância para a agilidade e qualidade de práticas administrativas. Cavalcante (2018) apresenta tendências da gestão pública brasileira, como a accountability, a participação e o engajamento social, o Governo Eletrônico e as tecnologias da informação e comunicação.

As transformações tecnológicas desafiam organizações a adaptarem-se às novas formas de gestão, reflexo da adaptação do trabalho e dos negócios frente às novas tecnologias. Momo, Schiavi, Behr e Lucena (2019) evidenciam isso no setor privado, onde o sucesso das empresas está relacionado não apenas com a introdução de novos produtos/serviços, mas com a inovação dos modelos de negócios. No setor público, esta lógica também é verdadeira, incentivando a criação de leis e regulamentos que guiem a implementação de políticas públicas e orientem processos administrativos com base na introdução de tecnologias a fim de que a administração pública se torne mais célere e ágil, correspondendo às novas demandas sociais e incrementando a democracia no país (Vieira \& Santos, 2010). Observa-se igualmente que a relação governo-cidadão tem gradualmente mudado, passando de centrada no governo para centrada no cidadão, valorizando assim a perspectiva de valor público.

Conforme Cepik, Canabarro e Possamai (2014), tecnologias da Era Digital como a Internet "modificam radicalmente a natureza e o modo de funcionamento da democracia, do governo e do próprio Estado" (p. 12). Assim, percebe-se a importância em manter atualizados estudos e pesquisas das novas tecnologias na gestão pública, a fim de proporcionar uma reflexão equilibrada das mudanças em evolução, buscando alimentar a pauta dos gestores governamentais na incorporação de questões acerca do uso e aplicação de novas tecnologias.

Esta crescente pauta tecnológica tem impulsionado a aceleração de mudanças institucionais. Para Schwab (2016), uma Quarta Revolução Industrial teve início na virada do século, baseada na Revolução Digital, cujas transformações são sentidas na economia, sociedade e governos. Uma tecnologia expoente é a Blockchain, a qual tem sido avaliada como uma ferramenta capaz de conferir confiabilidade e segurança aos dados. O pontochave está em sua cadeia de programação, onde a cada transação efetuada, uma chave criptografada única é gerada a partir de uma rede de verificação de aceitabilidade do código, tornando a transação segura e irreversível (Swan, 2015).

A Blockchain, que já é uma tecnologia em desenvolvimento em diversos setores privados (Momo, Schiavi, Behr, \& Lucena, 2019), também influenciará as instituições públicas e suas formas de gestão. Nesta perspectiva, a gestão da informação e a confiabilidade dos dados governamentais são essenciais para um diálogo mais próspero e construtivo entre sociedade e Estado (Cavalcante, 2018), sendo esta tecnologia determinante para proporcionar a confiabilidade de dados. Isto traz desafios à administração pública, como os relacionados com o bem-estar social, com o segundo setor e governos em si. Gestores públicos devem adaptar as formas de governança às novas tecnologias, tornando as estruturas do setor mais eficientes e com processos mais transparentes, mantendo os governos competitivos a nível global (Shava \& Hofisi, 2017).

Conforme Bindu, Sankar e Kumar (2019), estudos em governos eletrônicos trazem questões sobre dados abertos, redes de relacionamentos sociais, governança participativa e aplicações da Web 2.0 (transação de informações), demonstrando que a correlação governo-sociedade tem caminhado para uma democracia baseada em processos eletrônicos de tomada de decisão e troca de informações. A Blockchain é uma tecnologia que pode proporcionar segurança no armazenamento e gerenciamento de dados públicos, o que permite potencializar a justificativa da sua adoção. Przeybilovicz, Cunha e Meirelles (2018), ao tratar o uso das Tecnologias da Informação e Comunicação (TIC) para o desenvolvimento do Governo Eletrônico, identificam que é necessário considerar o contexto legal e socioeconômico onde se trabalha essa implementação, considerando os atores envolvidos e a capacidade institucional dos órgãos participantes. Também, deve-se considerar a necessidade de educação dos cidadãos no uso das tecnologias, facilitando práticas de democracia digital.

A necessidade de buscar a implementação do Governo Eletrônico, introduzindo a tecnologia nos processos públicos, visa melhorar a eficiência da prestação de serviços públicos juntamente com segmentação dos usuários desses serviços para responder melhor às demandas, mensurar com maior exatidão a efetividade das políticas públicas, responder às demandas econômicas do país e engajar 
os cidadãos nas decisões e políticas públicas a partir da confiança no setor gerada pela utilização de tecnologia (Al-Jenaibi, 2015).

Assim, considerando que o debate entre Blockchain e administração pública ainda está em fase de amadurecimento, este artigo busca responder: Quais as possíveis aplicações e impactos que a utilização da Blockchain pode proporcionar à administração pública?. A partir deste questionamento, e visando contribuir para o debate da transformação da Gestão Pública na Quarta Revolução Industrial, objetivase entender quais são as possíveis aplicações e consequências da utilização da Blockchain para a administração pública, optando-se por uma Revisão Sistemática (RS) como procedimento metodológico, seguindo o modelo PRISMA (Moher, Liberati, Tetzlaff, Altman, \& The PRISMA Group, 2015) para sua execução. Após esta introdução, a seção 2 traz o conceito da Blockchain, seguida da seção 3 que apresenta as etapas da RS e resultados. Na seção 4 apresenta-se uma análise crítica dos resultados, seguida da conclusão.

\section{BLOCKCHAIN}

A Blockchain é uma tecnologia com potencial de transformar a maneira como o mercado e os governos operam. Considerando suas características de confiabilidade, imutabilidade, autenticidade e auditabilidade, a partir da geração de dados criptografados que passam por uma rede de validação horizontal (Han, 2017), a tecnologia tem estimulado uma variedade de pesquisas que propõem sua utilização em diversos campos do conhecimento. Lançado em 2015 pelo governo do Reino Unido, o relatório chamado Distributed Ledger Technology: beyond blockchain aponta a importância da tecnologia e a considera uma possibilidade para a melhoria de serviços prestados pelo governo, indústria e serviços como: serviços financeiros, investimentos imobiliários, saúde e gerenciamento de identidade (Government Office for Science, 2016).

A Blockchain é baseada num algoritmo matemático que, através de uma corrente de blocos, identifica uma transação realizada virtualmente. A cadeia de blocos formada após a operação fica registrada e replicada em diversos servidores responsáveis por validar, por consenso, o registro. Isso torna essa criptografia segura, pois com diversas cópias espalhadas fica difícil alterar os blocos através de um ataque hacker (Government Office for Science, 2016). Essa tecnologia se baseia no conceito de Distributed Ledger Technology (DLT): um livro-razão distribuído. Swan (2015) define Blockchain:

The blockchain is a worldwide decentralized public ledger for the registration, acknowledgement, and transfer of all assets and societal interaction, a society's public records bank, an organizing mechanism to facilitate large-scale human progress in previously unimagined ways. ... The blockchain is a consensus model at scale, and possibly the mechanism we have been waiting for that could help to usher in an era of friendly machine intelligence (Swan, 2015, p. 94).

Esta tecnologia se popularizou através da sua implementação em um sistema mundial de transações financeiras realizado por meio da criptomoeda digital Bitcoin, idealizada por Nakamoto (2008). A cada transação financeira, uma chave criptografada é gerada, tornando a transação segura. Para validação, esse código passa por uma série de servidores, os quais o validarão através da mineração. Este procedimento objetiva resolver o valor do novo algoritmo criado quando uma nova transação é solicitada (Oberhaus, 2017), proporcionando a criação de um código criptografado único, inviolável, imutável e resiliente, capaz de promover uma transação financeira segura, rápida e sem intermediários (Zachariadis, Hileman, \& Scott, 2019).

O destaque do Bitcoin está na forma em que a Blockchain foi aplicada, atribuindo a ela uma série de características versáteis e inovadoras, contribuindo para sua popularização. Trata-se de uma tecnologia baseada em chave criptografada, uma sequência de blocos onde cada um deles possui um número pré-definido e sua união com outros blocos se dá a partir de uma lógica matemática. Assim, a sequência não é formada aleatoriamente, garantindo confiabilidade ao processo. Cada bloco representa uma operação, assegurada por assinaturas digitais criptografadas, significando que quem emite e quem recebe a transação estão protegidos, assim como o registro, conferindo transparência e accountability às transações (Formigoni Filho, Braga, \& Leal, 2017; Government Office for Science, 2016; Swan, 2015; Zachariadis et al., 2019).

A Blockchain conta igualmente com uma rede distribuída para verificação da autenticidade da operação e há uma cópia pública registrada em cada servidor que efetua a operação para cada fato realizado (Zachariadis et al., 2019). Adicionalmente, a transação entre pares agiliza diversos tipos de processos, eliminando intermediadores do processo (Formigoni Filho et al., 2017).

Considerando os atributos mencionados, observam-se características derivadas, como segurança no armazenamento dos registros, possibilitando a imutabilidade dos dados e, com isso, integridade e confiabilidade; descentralização das operações, criando uma rede de validação dificultando fraudes; possibilidade de uma accountability mais precisa e acessível, com operações registradas em 
um livro-razão distribuído imutável e criptografado (Nofer, Gomber, Hinz, \& Schiereck, 2017; Swan, 2015; Zachariadis et al., 2019). Contudo, cabe salientar que nem todo o funcionamento da Blockchain é, necessariamente, semelhante ao Bitcoin.

As redes Blockchain podem diferenciar-se em redes públicas (não-permissionadas) ou privadas (permissionadas). A primeira possui regras próprias, funcionando independente de aspectos legais ou regulatórios - como é o caso do Bitcoin; os validadores das transações são anônimos e a entrada para participar da rede de "mineradores" é de livre acesso. Já as privadas seguem regulamentação e os participantes são pré-selecionados, as aplicações ficam restritas a corporações fechadas (Formigoni Filho et al., 2017; Yermak, 2017). Ou seja, as chaves criptografadas de acesso para realização das operações em uma rede aberta são de amplo acesso e anônimas, enquanto em uma rede permissionada, as chaves de acesso são controladas e há a necessidade de solicitar permissão para as transações (Blockgeeks, 2019).

Conforme Pilkington (2016), pode-se pensar a essência do Blockchain como informacional e processual, e não apenas relacionada com a esfera monetária. Assim, considerando que a Blockchain pode ser pensada para além das transações financeiras, foi criada, por exemplo, a plataforma Ethereum ${ }^{1}$, que utiliza a Blockchain e permite a existência de um livro-razão seguro para viabilizar transações descentralizadas e generalizadas (Wood, 2014). Neste conceito, diversos tipos de transações podem ser realizados, desde a criação de tokens ou moedas próprias para cada empreendimento até a realização de smart contracts, transações mais complexas asseguradas pelas propriedades da Blockchain.

Os smart contracts são programações que combinam protocolos computacionais da interface dos usuários para executar termos de um contrato (Szabo, 1997). Com a Blockchain, o processo se torna simplificado em relação à tecnologia existente na época em que o conceito dos contratos inteligentes foi criado (Blockgeeks, 2017). Esta inovação pode, eventualmente, substituir a necessidade de advogados e bancos envolvidos em contratos de ativos, conforme se apresentam os termos do negócio entre ambas as partes; também, pode controlar os donos das propriedades, tangíveis (casas, automóveis) ou intangíveis (compartilhamentos de dados, permissão de acesso) (Nofer et al., 2017). Todas as possibilidades tornam-se viáveis tendo em vista a desobrigatoriedade de uma terceira parte para confirmar ou oportunizar o processo.

Beck, Avital, Rossi e Thatcher (2017) indicam prováveis tópicos de estudo relacionados à Blockchain, como plataformas de pagamentos, questões legais, implicações organizacionais, Internet das Coisas (IoT), desenvolvimento social e ambiental sustentável, eficiência energética, além de novas moedas e atores envolvidos nos futuros modelos organizacionais derivados da nova tecnologia. Colchester (2019) prevê tendências geradas pelo uso da Blockchain, como agências reguladoras governamentais mais flexíveis e iniciativas prontas para serem trabalhadas e lideradas por governos e consórcios mais transparentes, considerando os modelos de governança baseados em dados compartilhados e sistemas distribuídos.

Alcantara, Rodrigues, Lima e Nunes (2019) destacam iniciativas a nível governamental de uso da Blockchain, como a criação de plataforma de assistência médica no governo da Estônia, a iniciativa Dubai Blockchain Strategy, que pretende viabilizar transações financeiras através da Blockchain, além de almejarem que Dubai seja referência mundial no uso desta tecnologia. Também, os autores destacam a iniciativa holandesa Dutch Blockchain Action, uma agenda para o desenvolvimento da Blockchain que tem como pilares "a Identificação Digital; as Condições para o Uso da Tecnologia Blockchain; e a Capacitação para Desenvolvimento e Uso da Tecnologia" (Alcantara, Rodrigues, Lima, \& Nunes, 2019, p. 14).

Allessie, Sobolewski, Vaccari e Pignatelli (2019) apresentam um levantamento de projetos de aplicação da Blockchain na administração pública europeia, envolvendo uso em serviços notariais, bases distribuídas e smart-contracts para registro e transferência de propriedades de terras, certidões acadêmicas, pagamentos governamentais, sistema descentralizado de identidades. Os autores identificaram que a maioria dos projetos ainda estão em fase de teste, não garantindo resultados conclusivos sobre impactos na gestão pública. Os autores pontuaram que projetos que envolvem uma menor multiplicidade de stakeholders e uma governança mais centralizada possuem menor complexidade, tais como os projetos de aplicações notariais. Por outra via, projetos com soluções mais disruptivas envolvendo bases distribuídas e smart contracts, por exemplo, encaram mais desafios na implementação, geralmente relacionados a incompatibilidade com os processos administrativos em operação e não conformidade com regulações vigentes.

Também no Brasil, várias iniciativas têm sido concretizadas para apreciar a tecnologia e suas aplicações. Seguindo ainda em relação aos smart contracts, Luciano (2018) propôs sua utilização para a automação da gestão de contratos no processo de comercialização do gás natural brasileiro, mostrando as potenciais melhorias de controle referentes à contratos de empresas estatais.

Em janeiro de 2019, no seminário internacional Disruptive Technologies for Financial and Public Services no Banco Nacional de Desenvolvimento (BNDES), foi destacada a importância da transparência e da integridade dos dados e a Blockchain foi 
apresentada como uma das tecnologias fundamentais para tal transformação. Gladstone Arantes (analista de sistemas e líder técnico da Iniciativa Blockchain no BNDES), vê nessa tecnologia a possibilidade de alcançar os Objetivos do Desenvolvimento Sustentável da Organização das Nações Unidas (Agência BNDES de Notícias, 2019). Um dos projetos é utilizar a Blockchain para rastrear o dinheiro público de financiamento de projetos através da utilização de token (Leal, 2017).

O SERPRO lançou, em 2017, uma plataforma Blockchain para solucionar questões do Tesouro Direto (TD), com o objetivo de facilitar o acesso de qualquer cidadão ao investimento em títulos públicos do governo sem necessidade de possuir uma conta bancária (Serviço Federal de Gerenciamento de Dados [SERPRO], 2017).

Em espaços acadêmicos, cita-se o exemplo da Universidade Federal da Paraíba, que em fevereiro de 2019 entregou os primeiros diplomas digitais baseados em Blockchain. Esta iniciativa foi incentivada tendo em vista o alto índice de denúncias de diplomas falsificados e da falta de capacidade de comprovação da autenticidade dos comprovantes de ensino superior. A expectativa é que a tecnologia seja repassada para outras Instituições de Ensino Superior no país (Rede Nacional de Ensino e Pesquisa, 2019).

Apesar do otimismo frente ao desenvolvimento desta nova tecnologia, deve-se destacar que a implementação da Blockchain apresenta desafios e limitações técnicas, como a baixa capacidade de transferência de informações por segundo, o tempo de latência para gerar uma transferência segura, o tamanho dos dados gerados, a segurança, o desperdício de recursos energéticos utilizados para minerar, a dificuldade de criar programas que utilizam a Blockchain devido a sua complicada interface de programação de aplicações e o uso de versões mais simples de chaves criptográficas, que podem ser mais vulneráveis a ataques hackers (Yli-Huumo, Ko, Choi, Park, \& Smolander, 2016).

A partir do exposto, percebe-se que a Blockchain e a administração pública possuem uma relação positiva e construtiva. Também, é possível inferir que esta tecnologia afeta a dinâmica social, produtiva e organizacional através de novas ferramentas criadas para solucionar as problemáticas da contemporaneidade. Na próxima seção, através da realização de uma RSL utilizando o método Prisma, buscar-se-á explorar e apresentar esta relação.

\section{REVISÃO SISTEMÁTICA: BLOCKCHAIN NA ADMINISTRAÇÃO PÚBLICA}

A pesquisa desenvolvida valeu-se da Revisão Sistemática. Este método foi escolhido por objetivar o resumo de uma ampla quantidade de informações existentes sobre um fenômeno. Segundo Sampaio e Mancini (2007), "as revisões sistemáticas permitem incorporar um espectro maior de resultados relevantes, ao invés de limitar as conclusões à leitura de somente alguns artigos" (p. 84). Para Mendes-daSilva (2019), "entende-se que a publicação de revisões pode constituir um canal para publicação continuada de contribuições relevantes" (p. 4). Sendo assim, a partir da RS realizada, buscou-se colaborar com o estudo da Blockchain e potenciais aplicações no setor público. Para operacionalizar esta revisão, foram aplicadas as principais premissas do método PRISMA - Preferred Reporting Items for Systematic Reviews and Meta-Analyse (Moher et al., 2015), conforme exposto a seguir.

\section{As etapas da RS}

Nesta pesquisa, as atividades realizadas para a RS se baseiam nas orientações do método PRISMA (Moher et al., 2015). Para sintetizar a literatura a respeito da temática do artigo, bem como para contribuir para uma agenda de pesquisa futura (Mendes-Da-Silva, 2019), foram adotados aspectos recomendados pelo modelo PRISMA (Moher et al., 2015), especialmente os critérios de elegibilidade, análise e comunicação da pesquisa aplicáveis às revisões sistemáticas.

A RS iniciou pela (1) formulação da pergunta, seguida da (2) localização dos estudos e detalhamento da busca. Posteriormente, procedeu-se à (3) avaliação crítica dos estudos, com criação de filtros para selecionar os trabalhos relevantes e posterior (4) coleta de dados. Para finalizar, é feita uma (5) análise e apresentação dos dados, extraindo informações principais, para (6) interpretação dos dados e posterior (7) análise crítica, fonte de debate sobre o tema.

A pergunta-guia (1) da RS foi: Quais as possíveis aplicações e impactos que a utilização da tecnologia Blockchain pode proporcionar à administração pública?. Para responder à questão, a estratégia de busca dos estudos (2) valeu-se da definição da string de busca, com palavras-chave com capacidade de apresentar artigos correspondendo à investigação. Realizou-se um teste para verificar a quantidade de retornos de cada string elaborada. Na Tabela 1 estão apresentados os resultados obtidos na primeira etapa. Concomitantemente à definição das strings, foram escolhidas as fontes de busca: Web of Science, Scopus, Science Direct e Social Science Research Network (SSRN). 
Tabela 1. Strings de busca e quantidade obtida.

\begin{tabular}{|c|c|c|c|c|c|}
\hline Teste & String & Web of Science & Scopus & Science Direct & SSRN \\
\hline 1 & "administration" + "blockchain" & 24 & 59 & 289 & 19 \\
\hline 2 & $\begin{array}{c}\text { "public administration" + } \\
\text { "blockchain" }\end{array}$ & 8 & 18 & 43 & 6 \\
\hline 3 & $\begin{array}{c}\text { "public administration" + } \\
\text { "blockchain"+ "electronic } \\
\text { government" }\end{array}$ & 1 & 1 & 4 & 0 \\
\hline 4 & $\begin{array}{c}\text { "blockchain" + "electronic } \\
\text { government" }\end{array}$ & 11 & 2 & 5 & 11 \\
\hline 5 & $\begin{array}{c}\text { "public administration" + } \\
\text { "blockchain" + "applications" }\end{array}$ & 4 & 6 & 37 & 1 \\
\hline 6 & $\begin{array}{c}\text { "public administration" + } \\
\text { "blockchain" + "e-government" }\end{array}$ & 1 & 3 & 10 & 4 \\
\hline 7 & $\begin{array}{c}\text { "blockchain" + "government" } \\
\text { + "public management or } \\
\text { administration" }\end{array}$ & 6 & 6 & 109 & 5 \\
\hline
\end{tabular}

A

string selecionada foi "blockchain"+"government"+"public management or administration", pois, apesar de não ter apresentado sempre os maiores resultados quantitativos de retorno, obteve-se um resultado mais qualificado em função do tema em pesquisa, ou seja, a aplicação da Blockchain na administração pública ou correlacionada a esta. A Tabela 2 apresenta a forma que a busca foi realizada em cada plataforma.

Tabela 2. Formas de busca em cada plataforma.

\begin{tabular}{ll}
\hline Plataforma & Formato utilizado \\
\hline Scopus & Blockchain and government and public (management or administration) \\
Web of Science & TS=(blockchain AND government AND public AND (administration OR management)) \\
SSRN & blockchain government public ${ }^{*}$ \\
Science Direct & Blockchain AND government AND public AND (management OR administration) \\
\hline
\end{tabular}

Nota. *Após a realização da pesquisa principal, foi refinada a busca com as palavras management ou administration.

Foi realizada a criação de filtros para seleção dos artigos (3) que trariam informações mais relevantes ao estudo (Tabela 3). Excluiu-se inicialmente os artigos anteriores a 2013, pois percebeu-se que trabalhos anteriores à data traziam discussões mais tecnológicas e de base conceitual da Blockchain. Já os datados depois de 2013 traziam informações de aplicações e visões mais consolidadas de uso, estando mais em acordo com o objetivo desta pesquisa. O segundo filtro foi a seleção por idioma (inglês e espanhol) e o terceiro, por produção literária. Foram selecionados apenas artigos científicos, incluindo os de revisão; capítulos de livros e outras formas textuais foram desconsideradas.

Tabela 3. Filtros utilizados para a seleção dos artigos da RSL e sua justificativa.

\begin{tabular}{ccc}
\hline Ordem & Filtro & \multicolumn{1}{c}{ Justificativa } \\
\hline $1^{\circ}$ & Data de publicação & $\begin{array}{l}\text { O estudo da Blockchain para além das transações financeiras é uma novidade. Em anos } \\
\text { anteriores, os artigos versavam sobre a tecnologia em si. A partir de 2013, a area começou a } \\
\text { se consolidar e alguns estudos de caso foram relatados. }\end{array}$ \\
$2^{\circ}$ & Idioma & $\begin{array}{l}\text { As buscas foram realizadas com strings com termos em inglês. Artigos com resumos em } \\
\text { inglês, mas com texto em língua espanhola também foram aceitos. }\end{array}$ \\
$3^{\circ}$ & Seleção dos textos & $\begin{array}{l}\text { Foram selecionados apenas artigos científicos; livros e capítulos de livros foram excluídos, } \\
\text { bem como artigos repetidos. }\end{array}$ \\
$4^{\circ}$ & Análise dos resumos & $\begin{array}{l}\text { A partir dos resumos, observou-se a palavra "Blockchain". Caso o resumo apresentasse no } \\
\text { seu corpo este termo, então procurou-se por: Public Administration, Public Politics, Electronic } \\
\text { Government e E-Government. } \\
\text { Ao final do processo restaram 28 artigos, os quais foram lidos por inteiro e selecionados } \\
\text { apenas os que versavam especificamente sobre os temas administração pública e Blockchain. }\end{array}$ \\
\hline $5^{\circ}$ & Leitura dos artigos &
\end{tabular}


No quarto filtro foi realizada a seleção dos artigos. Os critérios de elegibilidade foram (i) a identificação da palavra Blockchain explícita no resumo e (ii) termos que remetessem ao universo que abrange a administração pública, tais como: policy, public policy, government, e-government, politics, public. Também, da relevância que a administração pública possuía em relação à proposta de uso da Blockchain.
Ao final, conforme Figura 1, restaram 28 artigos, os quais foram lidos para verificar se abrangiam o tema escolhido: Blockchain e administração pública. Destes, restaram 16 artigos para análise. As etapas 4, 5, 6 e 7 - coleta de dados, análise e apresentação dos dados, interpretação dos dados e análise crítica, respectivamente - serão apresentadas e discutidas nas seções seguintes. Os itens 5 e 6 foram realizados no programa Nvivo 12 Pro.

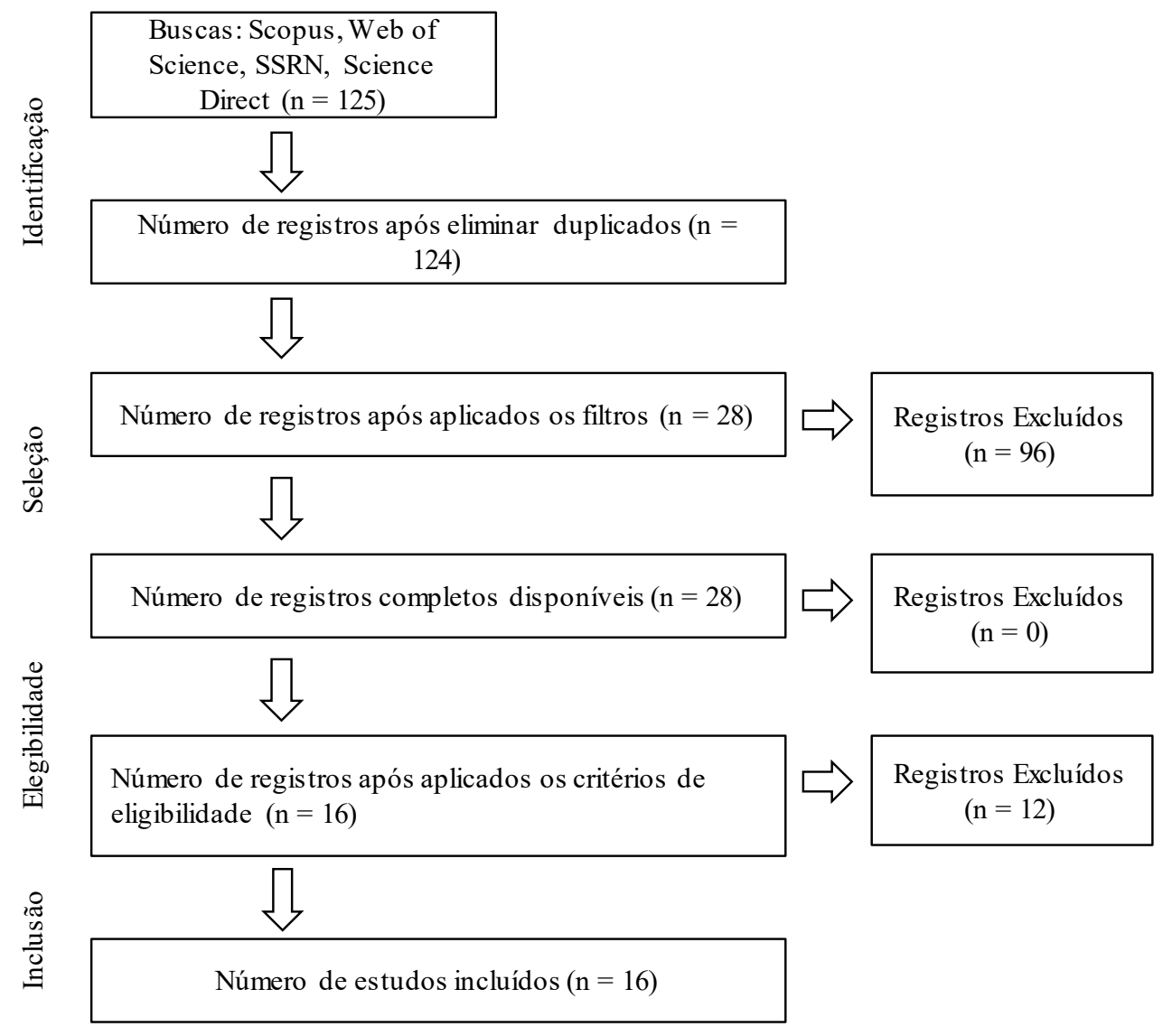

Figura 1. Fluxo do processo de seleção.

De acordo com o Modelo PRISMA de Moher D., Liberati A., Tetzlaff, J., Altman D. G., \& The PRISMA Group (2015). Principais itens para relatar revisões sistemáticas e meta-análises: A recomendação PRISMA. Epidemiologia e Serviços de Saúde, 24(2), pp. 355-342. Retrieved from http://www.scielo.br/ pdf/ress/v24n2/2237-9622-ress-24-02-00335.pdf

\section{Resultados da RSL}

Os resultados obtidos corroboraram com a percepção de que os estudos da Blockchain relacionados com a administração pública ainda são incipientes dentro do universo acadêmico. Entretanto, obteve-se um conjunto satisfatório das principais sugestões de aplicação da
Blockchain bem como dos potenciais impactos que estas poderiam gerar (Tabela 4). Apesar das aplicações da tecnologia não estarem suficientemente maduras e amplamente testadas, os achados foram contundentes com a proposta da pesquisa. 
Tabela 4. Coleta de dados: resumo das principais aplicações e potenciais impactos encontrados nos artigos analisados.

\begin{tabular}{|cccc|}
\hline Artigo & Autor(es)/Ano & Periódico & Aplicação Sugerida \\
$\begin{array}{c}\text { Algorithmic Government: } \\
\text { Automating Public Services and } \\
\text { Supporting Civil Servants in } \\
\text { using Data Science Technologies }\end{array}$ & $\begin{array}{c}\text { Engin e } \\
\text { Treleavenm } \\
(2019)\end{array}$ & $\begin{array}{c}\text { The Computer } \\
\text { Journal }\end{array}$ & $\begin{array}{c}\text { Smart Contracts; Gerenciamento de } \\
\text { contratos; Gestão de identidades; } \\
\text { Armazenamento de dados } \\
\text { governamentais }\end{array}$ \\
$\begin{array}{c}\text { Beyond the Hype: Distributed } \\
\text { ledger technology in the field of } \\
\text { public administration }\end{array}$ & $\begin{array}{c}\text { Kossow e Dykes } \\
(2018)\end{array}$ & Research Gate & $\begin{array}{c}\text { Segurança de Dados; Processamento } \\
\text { de Dados; Execução de pagamentos } \\
\text { governamentais; Identidade digital; } \\
\text { Votação }\end{array}$ \\
$\begin{array}{c}\text { Blockchain Governance and The } \\
\text { Role of Trust Service Providers: } \\
\text { The TrustedChain Network }\end{array}$ & $\begin{array}{c}\text { Atzori } \\
(2017)\end{array}$ & $\begin{array}{c}\text { Social Science } \\
\text { Research Network } \\
\text { (SSRN) }\end{array}$ & E-Governo; Administração Pública \\
\hline
\end{tabular}

\section{Blockchain in government: Benefits and implications of distributed ledger technology for information sharing \\ Blockchain in the built environment and construction industry: A systematic review, conceptual models and practical use cases}

Ø

\begin{abstract}
Blockchain meets Genomics: governance considerations for
promoting food safety and public health
\end{abstract}

\section{Blockchain = less government, more market}

\section{Blockchain Tracking and}

Cannabis Regulation: Developing

network to track Canada's

cannabis supply chain

El auge de blockchain y sus possibilidades reales de aplicación en los registros de las administraciones públicas

\section{Governance on the Drug Supply} Chain via Gcoin Blockchain

\section{Land records on Blockchain \\ for an implementation of Land Titling in India}

\section{Next Generation Government \\ Service Bus: The Blockchain Landscape}

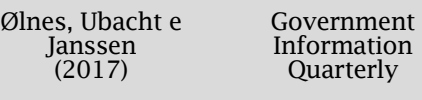

Reorganização dos processos organizacionais para que o serviço público se adapte à utilização da Blockchain

Utilizar a Blockchain dentro dos de cidades e governos inteligentes (2018)

$\begin{array}{cc}\begin{array}{c}\text { Abelseth } \\ (2018)\end{array} & \begin{array}{c}\text { Dalhousie Journal } \\ \text { of Interdisciplinary } \\ \text { Management }\end{array} \\ & \begin{array}{c}\text { Revista de } \\ \text { Maza }\end{array} \\ \begin{array}{c}\text { Internet,Derecho y } \\ \text { Política }\end{array}\end{array}$

SSRN alimentos na busca de patógenos

\section{Smart Contracts, votação, pagamentos, financiamentos} regulamentada pelo governo da cannabis no Canadá

A Blockchain seria utilizada para o governamentais

$\begin{array}{cc}\begin{array}{c}\text { Tseng, Liao, } \\ \text { Chong e Liao } \\ \text { (2018) }\end{array} & \begin{array}{c}\text { International Jorna } \\ \text { of Environmental } \\ \text { Research and Publi } \\ \text { Administration }\end{array} \\ \begin{array}{c}\text { Thakur, Doja, } \\ \text { Dwivedi, Ahmad } \\ \text { e Khadanga } \\ \text { (2019) }\end{array} & \begin{array}{c}\text { International } \\ \text { Journal of } \\ \text { Information }\end{array} \\ \text { Management }\end{array}$

$\underset{(2018)}{\text { Marchionni }}$ SSRN

a permissioned blockchain processos de construção melhorando o compartilhamento de informações e contribuindo para o desenvolvimento

Johnson
(2019) $\quad \begin{gathered}\text { Journal of Food Law } \\ \text { and Policy }\end{gathered} \quad \begin{gathered}\text { Utilização da Blockchain para } \\ \text { controlar a cadeia de suprimento de } \\ \text { alimentos na busca de patógenos }\end{gathered}$

Berg, Markey-

Utilizar a Blockchain para armazenar os dados de produção e distribuição armazenamento de diversos registros

Promoting public deliberation in low truste environments Australian use cases

\section{Lander e Cooper (2017)}

SSRN

\begin{tabular}{|c|c|c|}
\hline $\begin{array}{l}\text { Regulation as both enabler } \\
\text { of technology use and global } \\
\text { competitive tool: The Gibraltar } \\
\text { case }\end{array}$ & $\begin{array}{l}\text { Scholl e Bolívar } \\
\text { (2019) }\end{array}$ & $\begin{array}{l}\text { Government } \\
\text { Information } \\
\text { Quarterly }\end{array}$ \\
\hline $\begin{array}{l}\text { The promisse of peer-to- } \\
\text { peer? The potential impact } \\
\text { of blockchain on the actor } \\
\text { configuration in the Dutch } \\
\text { eletricity system }\end{array}$ & $\begin{array}{l}\text { Buth, Wieczorek } \\
\text { e Verbong } \\
\text { (2019) }\end{array}$ & $\begin{array}{l}\text { Energy Research } \\
\text { and Social Science }\end{array}$ \\
\hline $\begin{array}{l}\text { The security and financial } \\
\text { implications of Blockchain } \\
\text { technologies: regulating } \\
\text { merging technologies in Canada }\end{array}$ & $\begin{array}{l}\text { Ducas e Wilner } \\
\text { (2017) }\end{array}$ & $\begin{array}{c}\text { Canada's Journal } \\
\text { of Global Policy } \\
\text { Analysis }\end{array}$ \\
\hline
\end{tabular}

\section{Utilizar o sistema Blockchain Gcoin para proporcionar uma transação transparente de drogas} Utilização da Blockchain para registrar
as propriedades de terra e outros documentos relacionados

Utilização da Blockchain para aprimorar a comunicação entre os setores prestadores de serviço do governo

Utilização da Blockchain para votação
on line de projetos de políticas on line de projetos de políticas

\section{Deliberação da regulamentacão da Blockchain a partir dos stakeholders envolvidos}

Utilizar a Blockchain para armazenar os dados de geração de energia elétrica de pequena escala e permitir a venda e compra desta

Possibilidade de usar a regulamentação "sandboxes" para que as pesquisas com Blockchain possam se desenvolver públicas
Impactos Potenciais

Fomentar um governo tecnológico

a partir do fomento de startups, transformando os serviços públicos.

Segurança no armazenamento e processamento de dados; rastreabilidade das informações; transparência governamental

Evitar duplicidade de dados; Evitar duplicidade de dados;
integridade das informações; integridade das informaçôes;
efetividade na cobrança de taxas governamentais; melhoria do fluxo de trabalho

Desaparecimento de seções ou reestruturação/adaptação de órgãos intermediários governamentais a fim de garantirem o gerenciamento adequado ao funcionamento da Blockchain

Redução de burocracia e facilitação de transações financeiras

Parceria público-privada no gerenciamento do sistema (governança dos dados)

A partir da utilização da Blockchain diminuir a dependência das instituições governamentais

Regular e armazenar os dados referentes à produção da cannabis a fim de melhorar o controle de produção e diminuir a venda ilegal da droga

Maiores níveis de segurança e transparência de dados e redução de possíveis adulterações

Maior controle na distribuição de drogas, diminuindo o comércio de drogas falsificadas

Organização das estruturas e, consequentemente, do processo de registro de propriedade

Cada setor seria responsável pela sua gestão de dados e, através da Blockchain, trocariam informações precisas e de forma ágil

Garantia de transparência e confiabilidade à votação, tendo em vista a segurança de dados proporcionada pela Blockchain

Implementação da Blockchain como uma política pública que agregue valor público

Tornar viável, de forma inteligente, a utilização/implementacão de um mercado de energia local

Garantir segurança jurídico-legal para os testes em andamento que para os testes em andamento que utilizam a Blockchain, não sendo
restrito apenas a projetos pilotos governamentais 
A Figura 2 apresenta o gráfico de nuvem de palavras mais encontradas nos artigos analisados, ficando a palavra government em $5^{\circ}$ lugar.

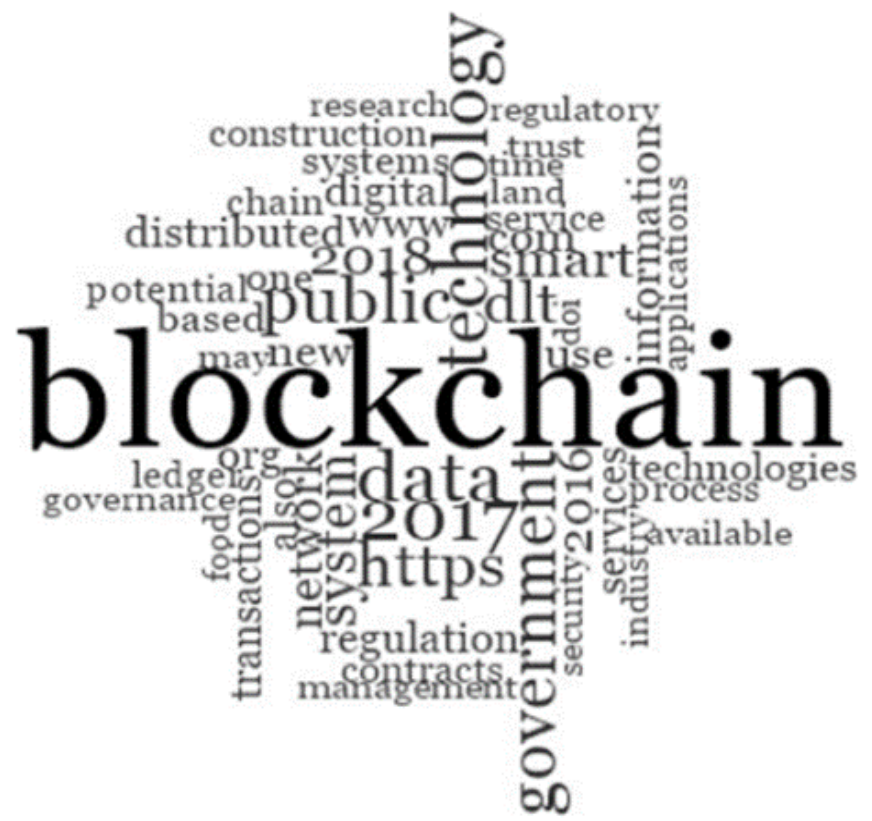

Figura 2. As 50 palavras mais encontradas nos artigos analisados.

As principais aplicações sugeridas foram consolidadas em quatro pontos principais: processamento de dados, segurança de dados, novos modelos de regulamentação estatal e procedimentos institucionais (Figura 3). Todas as possibilidades se firmam no mecanismo seguro de blocos criptografados que a Blockchain apresenta na sua programação, além de ter um sistema pautado na validação por nós do sistema para a confirmação de cada transação efetuada na rede, proporcionando confiabilidade às informações nela armazenadas.

Tendo em vista a forma distribuída que os dados são armazenados na blockchain, aplicações relacionadas a logística, propriedades de terra, energia elétrica, pagamentos governamentais e smart contracts acabam sendo adequadas, pois os diferentes atores envolvidos em cada processo se beneficiariam com o livro-razão distribuído (Atzori, 2018; Buth, Wieczorek, \& Verbong, 2019; Engin \& Treleaven, 2019; Kossow, 2019; Li, Greenwood, \& Kassem, 2019; Marchionni, 2018; Maza, 2019; Ølnes, Ubacht, \& Janssen, 2017; Thakur, Doja, Dwivedi, Ahmad, \& Khadanga, 2019). Votações, Cadeia de Suprimentos, Identidade Digital e Construção Civil partilham da eficiência do processamento de dados da Blockchain; a tecnologia se destaca pela segurança no armazenamento dos dados, pois são informações importantes, seja para o controle social - eleições e manutenção de documentos - ou para controle de construções e alimentos e/ou medicamentos, cabendo à administração pública controlar ou regulamentar esses processos (Abelseth, 2018; Johnson, 2019; Kossow, 2019; Lander \& Cooper, 2017; Li et al., 2019; Tseng, Liao, Chong, \& Liao, 2018).

Abordando uma questão mais estrutural, alguns achados apontaram para novos modelos de regulamentação estatal quando se trata sobre empreendimentos que utilizem a Blockchain como instrumento, utilizando o conceito de stakeholders para a deliberação regulamentadora (Scholl \& Bolívar, 2019) ou a aplicação do modelo sandboxes, que flexibiliza a legislação vigente para que empresas apliquem tecnologias avançadas, como a Blockchain, sem que sejam inibidas pela rigidez legal, mas ao mesmo tempo, fiscalizadas pelo poder público (Atzori, 2018). Também há propostas de mudanças mais radicais quanto aos processos institucionais, principalmente estatais, retirando destas o controle

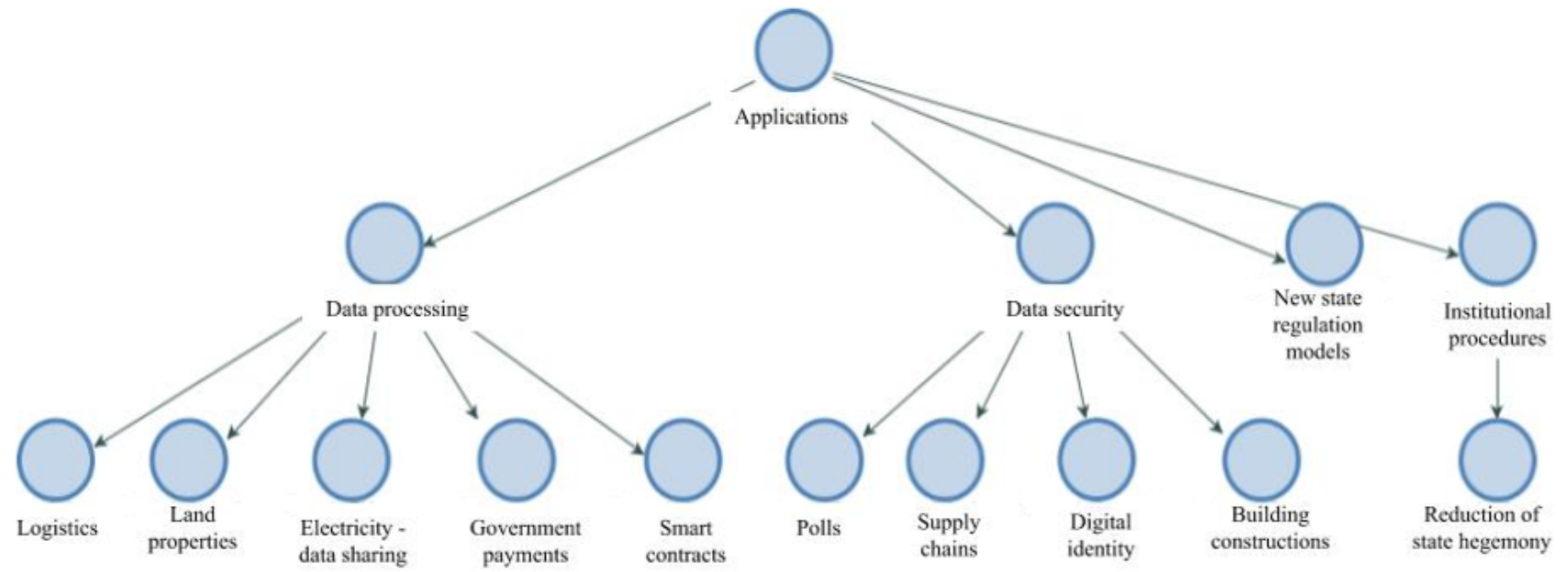

Figura 3. Principais achados sobre aplicações da Blockchain nos artigos analisados. 
principal (Berg, Markey-Towler, \& Novak, 2018); contudo, isso requer uma discussão aprofundada.

Dentre os impactos potenciais a partir dos resultados obtidos (Figura 4), expõe-se que a utilização da Blockchain leva à melhoria no armazenamento de dados, podendo acarretar na redução dos processos burocráticos, agilizando o processamento de dados e permitindo, por exemplo, uso de smart contracts. Além disso, apresenta-se como uma ferramenta importante para o compartilhamento de informações como dados clínico-patológicos, de distribuição de drogas e de etapas da cadeia de suprimentos de diversos produtos, contribuindo para uma fiscalização e controle mais eficientes (Abelseth, 2018; Atzori, 2018; Berg et al., 2018; Buth et al., 2019; Engin \& Treleaven, 2019; Johnson, 2019; Kossow, 2019; Li et al., 2019; Marchionni, 2018; Thakur et al., 2019; Tseng et al., 2018).

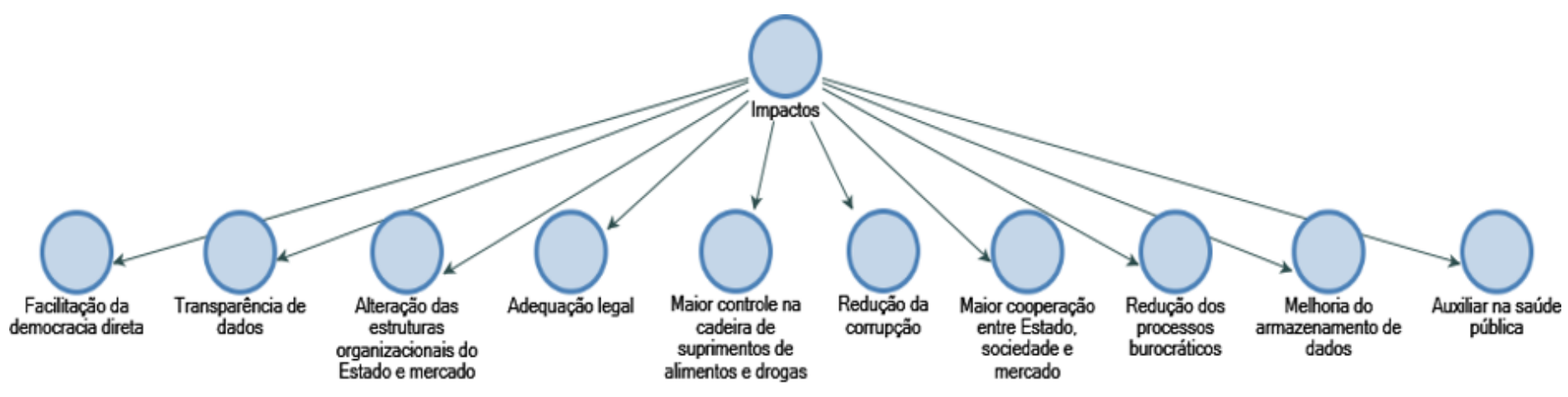

Figura 4. Principais achados sobre possíveis impactos da Blockchain nos artigos selecionados.

A identificação de que a Blockchain impactaria na facilitação da democracia direta e na transparência de dados foi verificada a partir da possibilidade de oferecer um novo mecanismo para a construção de políticas públicas e de participação social, considerando a possibilidade de eleições eletrônicas confiáveis (Berg et al., 2018; Lander \& Cooper, 2017). Valendo-se da rede distribuída, seria possível melhorar a confiabilidade e a facilidade de comunicação entre os atores envolvidos, permitindo um modelo bottom-up de construção de políticas públicas organizado e eficiente (Ducas \& Wilner, 2017). Também, essa nova tecnologia, por suas características, auxiliaria no combate à corrupção, trazendo mais celeridade à administração pública e confiabilidade no setor.

Outro impacto verificado foi a possível necessidade de alteração das estruturas organizacionais do Estado e do mercado em relação a este. Esta mudança teria como início a reorganização das estruturas públicas administrativas podendo chegar a uma reforma de Estado pois, com a nova dinâmica de funcionamento a ser proporcionada pela Blockchain - como a eficiência na gestão de dados - instituições, órgãos ou setores poderão tornar-se obsoletos, com necessidade de alterar atividade ou funcionalidade destes (Berg et al., 2018; Marchionni, 2018; Ølnes et al., 2017). Também se deve considerar que a utilização da tecnologia Blockchain demanda uma maior cooperação entre Estado, sociedade e mercado, haja vista que a tecnologia tem seu cerne no compartilhamento do banco de dados, gerando transparência e accountability, tornando imprescindível a construção de estruturas de diálogo permanente entre atores (Abelseth, 2018; Buth et al., 2019; Scholl \& Bolívar, 2019). Contudo, para implementação da Blockchain no contexto atual, é impreterível a adequação legal desta tecnologia às estruturas já vigentes (Abelseth, 2018; Engin \& Treleaven, 2019; Kossow, 2019; Scholl \& Bolívar, 2019; Ølnes et al., 2017), tendo em vista que Estados modernos tem como característica fundamental a legitimidade da aplicação da lei.

A partir dos resultados, verifica-se uma baixa incidência de estudos explorando aplicações da Blockchain na administração pública, a maioria deles identificados como estudos pilotos apresentando as potenciais áreas de atuação para a aplicação da Blockchain neste setor. Assim, após análise e apresentação dos dados obtidos (etapa 5), estes serão discutidos com maior profundidade na seção 4. 


\section{APLICAÇÕES DA BLOCKCHAIN E CONSEQUÊNCIAS POTENCIAIS}

Diversas são as possíveis aplicações e consequências que a tecnologia Blockchain pode trazer para a esfera pública, tanto nas estruturas e processos governamentais, quanto na relação sociedade-Estado para a formulação de políticas públicas e de participação social. A Tabela 5 apresenta um panorama comparativo entre as aplicações da Blockchain encontradas na RSL e os benefícios de seu uso para a administração pública.

Tabela 5. Comparação entre os resultados obtidos e sua aplicação na administração pública.

\begin{tabular}{|c|c|c|}
\hline Aplicações da Blockchain & Benefícios na administração pública & Princípios \\
\hline \multirow[t]{2}{*}{ Processamento de dados } & Combate a corrupção e transparência & $\begin{array}{l}\text { Armazenamento de bases de dados } \\
\text { distribuídas, melhorando a segurança dos } \\
\text { dados públicos, proporcionando melhor } \\
\text { gestão, transparência e imutabilidade }\end{array}$ \\
\hline & Serviços notariais sem fraudes & $\begin{array}{l}\text { Aplicada ao setor de registro de propriedades } \\
\text { de imóveis, evitando adulterações }\end{array}$ \\
\hline Segurança de dados & $\begin{array}{l}\text { Incremento dos mecanismos de participação } \\
\text { cidadã }\end{array}$ & $\begin{array}{l}\text { Eleições e/ou votações com suporte da } \\
\text { Blockchain para confiabilidade ao processo }\end{array}$ \\
\hline Novos modelos de regulamentação estatal & $\begin{array}{l}\text { Descentralização segura de registros civis e } \\
\text { outros dados utilizados pela administração } \\
\text { pública }\end{array}$ & $\begin{array}{l}\text { Processamento e armazenamento de dados } \\
\text { na Blockchain, proporcionando melhor } \\
\text { gestão, transparência e imutabilidade }\end{array}$ \\
\hline Novos procedimentos institucionais & $\begin{array}{l}\text { Eficiência, atualização e modernização de } \\
\text { processos e regulações do setor público }\end{array}$ & $\begin{array}{l}\text { Reestruturação das instituições públicas } \\
\text { a fim de adaptação à implementação da } \\
\text { Blockchain, trazendo eficiência ao setor }\end{array}$ \\
\hline
\end{tabular}

A partir dos trabalhos explicitados verifica-se que a Blockchain apresenta a segurança de dados como um dos principais atributos, podendo ser utilizada em votações e processamento de dados de forma eficiente, evitando fraudes e duplicidades, ao garantir armazenamento em diversas cópias imutáveis espalhadas pela rede, garantindo accountability e transparência das informações. Assim, a Blockchain poderia auxiliar, também, no combate à corrupção, considerando a dificuldade de implementar e disseminar dados fraudulentos em plataformas que utilizam esta tecnologia. A partir disso, conclui-se que a Blockchain poderia ser uma ferramenta capaz de trabalhar em prol da sociedade, trazendo segurança na informação e garantindo o cumprimento da legislação, por meio do modelo de armazenamento de dados governamentais criptografados e dos livros-razão distribuídos. Conforme análise apresentada na seção 3.2, verificase que a Blockchain é um modelo tensionado para o armazenamento de dados que tratam sobre propriedade de terras e suas demarcações, permitindo a facilitação do processo de compra e registro de imóveis e/ou terras, além de maior transparência (Maza, 2019; Thakur et al., 2019). Disputas de propriedade ou demarcação de terras, sejam agrárias, quilombolas, indígenas ou urbanas e ainda não implementada em reforma agrária (Reis, 2012), poderiam ser simplificadas pela utilização da
Blockchain, na definição de titularidade de terras e imutabilidade, minimizando conflitos.

Outro ponto considerado chave é a participação cidadã. Segundo Teixeira (2008), um grande desafio para proporcionar a participação tem sido o acesso à informação. Para tanto, a Blockchain pode ser forte aliada na promoção de instrumentos que proporcionem esse acesso. Por exemplo, ao final de reuniões de conselhos de políticas públicas, suas deliberações poderiam ser votadas de forma anônima, inibindo formas de coerção interna, e o resultado poderia ser cruzado diretamente com o setor financeiro de atuação do conselho, gerando um dado concreto da necessidade pública e de viabilidade orçamentária. Também, essas decisões poderiam ser tomadas pela população, ou parte dela, através de um processo de votação em uma plataforma Blockchain, contribuindo para o incremento da democracia.

Além da segurança, a Blockchain é importante instrumento para armazenamento de dados e facilitação da gestão dos mesmos. Nesta perspectiva, a tecnologia também contribui na melhoria da abertura e disponibilização de dados, contribuindo para a transparência, uma das necessidades apontadas pela Diretoria de Análise de Políticas Públicas (DAPP) da FGV e a Open Knowledge Brasil (OKBR), que verificaram imperfeições quanto à abertura dos dados governamentais. Segundo 
Albuquerque (2017), um dos principais problemas encontrados foi a dificuldade em trabalhar com dados e sua não disponibilização completa para download.

As licitações são um mecanismo de contratação de serviços e de compras da administração pública que busca dar celeridade ao processo de escolha das empresas, a partir de um processo administrativo rígido de editais (no Brasil regido principalmente pela Lei n. 8666, 1993). Mesmo com toda a regulamentação, as fraudes licitatórias ainda são comuns. Por conseguinte, os smart contracts poderiam ser uma solução inovadora para o processo de contratação governamental. Este tipo de contrato, gerado dentro do sistema Blockchain, apenas é rodado quando uma série de critérios especificados na programação são validados pela rede para então efetivar a transação. Assim, seria viável verificar se a empresa a ser contratada é idônea, se o orçamento é adequado para a realização da compra, entre outras questões que garantiriam maior eficiência e accountability ao setor público (Luciano, 2018).

Apesar de viabilizar transações ainda consideradas lentas para sistemas computacionais, a Blockchain pode ser uma opção mais ágil em comparação ao que é oferecido atualmente para a gestão pública. A confecção de documentos, como carteira de identidade, de trabalho, de habilitação, certidão de nascimento, de casamento, entre outros, podem ter seu tempo reduzido com a Blockchain. Com a nova tecnologia, todos estes registros poderiam ser armazenados em um mesmo código, sendo de fácil acesso e confiável, tendo em vista a imutabilidade que a tecnologia oferece dos dados no sistema, corroborando com a perspectiva de utilização da Blockchain para registros públicos mais eficientes e transparentes (Maza, 2019). Além disso, experiências com identidades e outros documentos digitais com validação eletrônica já estão em testes, a nível governamental, mostrando importância de ter uma tecnologia que suporte essa evolução (Allessie, Sobolewski, Vaccari, \& Pignatelli, 2019).

Há também possibilidade de melhor gestão em processos eleitorais, especialmente no controle de segurança e agilidade no processo. A Blockchain poderia melhorar a confiabilidade dos votos, pois a geração dos dados eleitorais ficaria registrada em diversos servidores de forma a impossibilitar, na prática, qualquer tipo de alteração. Também, permitiria um sistema de votação mais dinâmico, podendo facilitar a condução de plebiscitos e consultas públicas (Lander \& Cooper, 2017). Assim, novas formas de gestão do bem público poderiam ser pensadas, diminuindo a influência direta e, muitas vezes, unilateral do Estado. Contudo, é importante relembrar que, para uma organização social harmônica, faz-se necessário canais de relacionamento que incluam atores interessados, envolvidos na política pública (Scholl \& Bolívar, 2019). Exemplos de oportunidades para novos modelos de regulamentação estatal estão no controle logístico de alimentos, drogas, energia elétrica, como visto anteriormente.

Com base no exposto, conclui-se que, para viabilizar a realização dessas mudanças e implementação desses benefícios, seria necessária a reorganização de estruturas governamentais e o remodelamento de processos internos, iniciando pela padronização do armazenamento de dados e buscando uma maior integração entre os órgãos de governo. Então, haveria a possibilidade de estabelecer um sistema realmente integrado e novas regulações, melhorando significativamente os serviços e processos do governo eletrônico (Berg et al., 2018; Marchionni, 2018; Ølnes et al., 2017).

\section{CONCLUSÕES}

As novas tecnologias, características da Quarta Revolução Industrial, influenciam a forma como a sociedade, as empresas e os governos se relacionam e se mantêm. A Blockchain, tecnologia considerada disruptiva, já é uma realidade. Entendê-la é mais do que necessário para que se esteja preparado para os novos rumos que a organizações e a sociedade tomarão.

No aspecto da gestão pública, o surgimento do Governo Eletrônico e da Parceria para Governo Aberto incentivaram políticas que intentam melhorar o relacionamento do administrador público e os cidadãos, buscando proporcionar maior transparência e participação popular através da exposição dos dados governamentais e melhorias nos serviços eletrônicos. Levando em consideração as novas tecnologias desenvolvidas no século XXI, a Blockchain impacta a forma de conceber processos, bem como no armazenamento de dados e na utilidade que as instituições intermediárias possuem dentro do sistema organizacional.

A pesquisa realizada demonstrou que o tema Blockchain e administração pública tem ganho relevância e importância conforme avança a disseminação desta tecnologia, pois foi verificada a elevação da ocorrência de artigos sobre o tema nos últimos anos. Além disso, com a RSL, foi possível traçar um panorama geral sobre as principais aplicações de Blockchain e seus impactos, evidenciando uma área de pesquisa em pleno desenvolvimento, com oportunidades de aplicação em diferentes setores.

Com base nos resultados obtidos, observouse que a Blockchain pode ensejar confiabilidade e 
qualidade na gestão dos dados governamentais, bem como celeridade na celebração de contratos da administração pública, contribuindo para inibir fraudes em licitações, e adulteração de documentos, notas de pagamentos, entre outros. Além disso, possibilitaria um mecanismo eficiente para processos eleitorais descentralizados, corroborando para um modelo mais participativo nas decisões das políticas públicas, com agilidade e anonimato. Também, seria uma ferramenta importante para o controle logístico de produtos que necessitam de regulamentação estatal. Mesmo com resultados dos estudos de iniciativa governamental da Blockchain ainda, em sua maioria, em níveis pilotos e experimentais, mantêmse a expectativa de aprofundar o conhecimento a fim de identificar soluções para problemas que fragilizam a democracia, como corrupção, burocratização e ineficiência.

A despeito das aplicações e impactos positivos demonstrados sobre a utilização da Blockchain na administração pública, deve-se atentar que a tecnologia não transforma a realidade por si só, mas oferece um novo instrumento à serviço da sociedade. A Blockchain, ao proporcionar a segurança dos dados, pode aprimorar a eficiência nos serviços da administração pública e fortalecer o exercício da participação democrática. Tal tecnologia viabiliza a horizontalidade, transparência e confiabilidade na gestão das informações, desde que sua instrumentalização seja feita de forma a servir aos interesses sociais, tornando-se um meio de atingir o desenvolvimento de ações construtivas e participativas. Para este fim, é indispensável o

\section{REFERÊNCIAS}

Abelseth, B. (2018). Blockchain tracking and cannabis regulation: Developing a permissioned blockchain network to track Canada's cannabis supply chain. Dalhousie Journal of Interdisciplinary Management, 14, 1-11. https://doi.org/10.5931/djim.v14i0.7869

Agência BNDES de Notícias (2019, janeiro 15). Blockchain: Especialistas discutem vantagens da tecnologia em serviços públicos e financeiros. Retrieved in February 20, 2019, from https://agenciadenoticias.bndes. gov.br/detalhe/noticia/Blockchain-Especialistasdiscutem-vantagens-da-tecnologia-em-servicospublicos-e-financeiros/ diálogo e a cooperação entre as partes envolvidas na construção da sociedade - universidades, cidadãos, empresas e governo.

Também, é preciso debater as características inerentes ao setor público, que responde de forma vagarosa às mudanças institucionais e tecnológicas. Conforme análise de Possamai (2014) baseada nos estudos de Fountain, é necessário pensar as TIC a partir do reconhecimento da existência de Mecanismos de Estabilidade Institucional, que se formam para dar segurança e continuidade ao processo gerencial estatal, e de variáveis sociopolíticas na construção de políticas públicas do governo eletrônico.

Assim, sugere-se para pesquisas futuras aprofundar os estudos sobre governo eletrônico a partir da análise da legislação pertinente ao assunto, buscando apreciar as variáveis sociológicas e políticas que corroboram para a introdução de mecanismos tecnológicos no governo, bem como a ampliação de fontes de busca para criar um referencial de apoio mais sólido sobre a tecnologia Blockchain, contribuindo para o debate sobre tecnologias na administração pública.

\section{Nota}

${ }^{1}$ Ethereum é uma plataforma descentralizada que executa contratos inteligentes: aplicativos que funcionam exatamente como programados, sem qualquer possibilidade de tempo de inatividade, censura, fraude ou interferência de terceiros.
Al-Jenaibi, B. N. (2015). The new electronic government: Are the federal authorities ready to use e-government? International Journal of Knowledge Society Research (IJKSR), 6(3), 45-74. https://doi.org/10.4018/ IJKSR.2015070104

Albuquerque, E. M. (2017). Open Knowledge Brasil e DAPP lançam índice de dados abertos para Brasil. Retrieved in July 18, 2018, from https://br.okfn.org/2017/04/27/openknowledge-brasil-e-dapp-lancam-indice-de-dadosabertos-para-brasil/

Alcantara, L. T. de, Rodrigues, E. A. S., Lima, D. V. de, \& Nunes, A. (2019). Uso da tecnologia Blockchain como instrumento de governança eletrônica no setor público. Trabalhos do Congresso Internacional de Contabilidade Pública, Lisboa, Portugal, 2. Retrieved from https://www.occ. pt/dtrab/trabalhos/iicicp//finais_site/67.pdf. 
Allessie, D., Sobolewski, M., Vaccari, L., \& Pignatelli, F. (Editor) (2019). Blockchain for digital government. Luxembourg: Publications Office of the European Union. https:// doi.org/10.2760/942739

Atzori, M. (2018). Blockchain governance and the role of trust service providers: The TrustedChain ${ }^{\circledR}$ network. The Journal of the British Blockchain Association, 1(1), 1-17. https://doi.org/10.31585/jbba-1-1-(3)2018

Beck, R., Avital, M., Rossi, M., \& Thatcher, J. B. (2017). Blockchain technology in business and information systems research. Business \& Information Systems Engineering, 59(6), 381-384. https://doi.org/10.1007/s12599-0170505-1

Berg, A., Markey-Towler, B., \& Novak, M. (2018). Blockchains $=$ less government, more market. Journal of Private Enterprise, Forthcoming, 1-12. https://doi. org/10.2139/ssrn.3301714

Bindu, N., Sankar, C. P., \& Kumar, K. S. (2019). From conventional governance to e-democracy: Tracing the evolution of e-governance research trends using network analysis tools. Government Information Quarterly, 36, 385399. https://doi.org/10.1016/j.giq.2019.02.005

Blockgeeks (2017). Smart contracts: The blockchain technology that will replace lawyers. Retrieved from https:// blockgeeks.com/guides/smart-contracts/

Blockgeeks (2019). What is blockchain technology? A step-by-step guide for beginners. Retrieved from https://blockgeeks.com/guides/what-isblockchain-technology/

Buth, M. C., Wieczorek, A. J., \& Verbong, G. P. J. (2019). The promise of peer-to-peer trading? The potential impact of blockchain on the actor configuration in the Dutch electricity system. Energy Research \& Social Science, 53, 194-205. https://doi.org/10.1016/j.erss.2019.02.021

Cavalcante, P. (2018). Innovations in the federal government during the post-new public management era. Revista de Administração Contemporânea, 22(6), 885-902. https://doi.org/10.1590/1982-7849rac2018170391

Cepik, M., Canabarro, D. R., \& Possamai, A. J. (2014). Do novo gerencialismo público à governança da era digital. In M. S. Pimenta \& D. R. Canabarro (Orgs.), Governança de TI - transformando a administração pública no Brasil. Porto Alegre: UFRGS/CEGOV.

Chahin, A., Cunha, M. A., Knight, P. T., \& Pinto, S. L. (2004). E-gov: a próxima revolução brasileira - eficiência, qualidade e democracia: O governo eletrônico no Brasil e no mundo. São Paulo: Prentice Hall.

Colchester, R. (2019). Mais 6 tendências de blockchain para 2019. Revista CIO, Tendências. Retrieved from https://cio.com.br/mais-6-tendencias-de-blockchainpara-2019/

Ducas, E., \& Wilner, A. (2017). The security and financial implications of blockchain technologies: Regulating emerging technologies in Canada. Canada's Journal of Global Policy Analysis, 72(4), 538-562. https://doi. org/10.1177/0020702017741909

Engin, Z., \& Treleaven, P. (2019). Algorithmic government: Automating public services and supporting civil servants in using data science technologies. The Computer Journal, 62(3), 448-460. https://doi. org/10.1093/comjnl/bxy082
Formigoni Filho, J. R., Braga, A. M., Leal, R. L. V. (2017). Tecnologia blockchain: Uma visão geral. Retrieved from https:// www.cpqd.com.br/wp-content/uploads/2017/03/ cpqd-whitepaper-blockchain-impresso.pdf

Government Office for Science (2016). Distributed ledger technology: Beyond block chain. Retrieved from https://assets.publishing.service.gov.uk/ government/uploads/system/uploads/attachment_ data/file/492972/gs-16-1-distributed-ledgertechnology.pdf

Han, G. E. (2017). Blockchain: the promise of smart contracts. Juris Illuminae, 8, 1-4. Retrieved from https://staticl.squarespace.com/ static/55c714fbe4b0f0d634b061b5/t/58922c3b1b63 1b5940ff0b4e/1485974587997/Goh+Eng+Han+-+Bloc kchain+the+promise+of+smart+contracts.pdf

Johnson, W. G. (2019). Blockchain meets genomics: Governance considerations for promoting food safety and public health. Journal of Food Law and Policy, 15(1), 74-97. Retrieved from https://scholarworks.uark.edu/jflp/ vol15/iss $1 / 3$

Kossow, N. (2019). Beyond the hype: Distributed ledger technology in the field of public administration. [Working Paper $\mathrm{n}^{0}$ 58]. European Research Centre for Anti-Corruption and State-Building Hertie School of Governance, Berlin, Germany.

Lander, L., \& Cooper, N. (2017, August). Promoting public deliberation in low trust environments: Australian use cases. CEUR Workshop Proceedings of the International Joint Conference on Artificial Intelligence, Melbourne, Australia, 26.

Leal, M. (2017). Governo brasileiro quer criar laboratório de Blockchain. Retrieved from https://www. criptofacil.com/governo-brasileiro-quer-criarlaboratorio-de-blockchain/

Lei n. 8666, de 21 de junho de 1993. (1993). Regulamenta o art.37, inciso XXI, da Constituição Federal, institui normas para licitações e contratos da Administração Pública. Diário Oficial da União. Brasília, DF: Presidência da República.

Li, J., Greenwood, D., \& Kassem, M. (2019). Blockchain in the built environment and construction industry: A systematic review, conceptual models and practical use cases. Automation in Construction, 102, 288-307. https://doi.org/10.1016/j.autcon.2019.02.005

Luciano, R. B. de S. (2018). Aplicação smart contract nos contratos de gás natural: Uma análise exploratória. Revista de Administração Contemporânea, 22(6), 903-921. https://doi.org/10.1590/1982-7849rac2018180136

Marchionni, P. (2018). Next generation government service bus: The blockchain landscape. Available from http:// dx.doi.org/10.2139/ssrn.3141749

Maza, M.V. (2019) El auge de blockchain y sus posibilidades reales de aplicación en los registros de las administraciones públicas. IDP. Revista de Internet, Derecho y Ciencia Política, (28), 109-126. http://doi. org/10.7238/idp.v0i28.3154

Melati, C., \& Janissek-Muniz, R. (2019, June). Smart government: análise de dimensões sob a perspectiva de Gestores Públicos. Anais do Encontro Brasileiro de Administração Pública, Salvador, BA, Brazil, 6.

Mendes-da-Silva, W. (2019) Contribuições e limitações de revisões narrativas e sistemáticas na área de negócios. Revista de Administração Contemporânea, 23(2), 1-11. https://doi.org/10.1590/1982-7849rac2019190094 
Moher D., Liberati A., Tetzlaff, J., Altman D. G., \& The PRISMA Group (2015). Principais itens para relatar revisões sistemáticas e meta-análises: A recomendação PRISMA. Epidemiologia e Serviços de Saúde, 24(2), 355-342. Retrieved from http://www.scielo.br/pdf/ ress/v24n2/2237-9622-ress-24-02-00335.pdf

Momo, F. S., Schiavi, G. S., Behr, A., \& Lucena, P. (2019). Business models and blockchain: What can change? Revista de Administração Contemporânea, 23(2), 228-248. https://doi.org/10.1590/19827849rac2019180086

Nakamoto, S. (2008). Bitcoin: A peer-to-peer eletronic cash system. Retrieved from https://bitcoin.org/bitcoin. pdf

Nofer, M., Gomber, P., Hinz, O., \& Schiereck, D. (2017). Blockchain. Business \& Information Systems Engineering International Journal, 59(3), 183-187. https://doi.org/10.1007/s12599-017-0467-3

Oberhaus, D. (2017). Um guia simplificado para montar seu próprio equipamento e 'farmar' Ethereum. Vice. Retrieved from https://motherboard.vice.com/ pt_br/article/59zjgq/um-guia-simplificado-paramontar-seu-proprio-equipamento-para-farmarethereum

Ølnes, S., Ubacht, J., \& Janssen, M. (2017). Blockchain in government: Benefits and implications of distributed ledger technology for information sharing. Government Information Quarterly, 34(3), 355-364. https://doi.org/10.1016/j.giq.2017.09.007

Pilkington, M. (2016). Blockchain technology: Principles and applications. In F. X. Olleros \& M. Zhegu (Eds.), Research handbook on digital transformations. Cheltenham, UK: Edward Elgar Publishing.

Possamai, A. J. (2014). Governo eletrônico: uma análise institucional. In M. S. Pimenta \& D. R. Canabarro (Orgs.), Governança digital. Porto Alegre: UFRGS/ CEGOV.

Przeybilovicz, E., Cunha, M. A., \& Meirelles, F. D. S. (2018). The use of information and communication technology to characterize municipalities: Who they are and what they need to develop e-government and smart city initiatives. Revista de Administração Pública, 52(4), 630-649. https://dx.doi.org/10.1590/00347612170582

Rede Nacional de Ensino e Pesquisa. (2019). UFPB realiza solenidade de entrega dos primeiros diplomas universitários digitais do país. RNP - Rede Nacional de Ensino e Pesquisa. Retrieved from https://www. rnp.br/noticias/ufpb-realiza-solenidade-de-entregados-primeiros-diplomas-universitarios-digitais-dopais

Reis, R. R. (2012) O direito à terra como um direito humano: A luta pela reforma agrária e o movimento de direitos humanos no Brasil. Lua Nova: Revista de Cultura e Política, (86), 89-122. https://dx.doi.org/10.1590/ S0102-64452012000200004

Sampaio, R. F., \& Mancini, M. C. (2007). Estudos de revisão sistemática: um guia para síntese criteriosa da evidência científica. Revista Brasileira de Fisioterapia, $11(1)$, 83-89. http://dx.doi.org/10.1590/S141335552007000100013

Schwab, K. (2016). A quarta revolução industrial (1st ed.). São Paulo: Edipro.
Scholl, H. J., \& Bolívar, M. P. R. (2019). Regulation as both enabler of technology use and global competitive tool: The Gibraltar case. Government Information Quarterly, 36(3), 601-613. http://dx.doi. org/10.1016/j.giq.2019.05.003

Serviço Federal de Gerenciamento de Dados (2017). Serpro lança plataforma blockchain. Retrieved from http:// www.serpro.gov.br/menu/noticias/noticias-2017/ serpro-lanca-plataforma-blockchain-2

Shava, E., \& Hofisi, C. (2017). Challenges and opportunities for public administration in the fourth industrial revolution. African Journal of Public Affairs, 9(9), 203-215. Retrieved from https://hdl.handle. net/10520/EJC-c13dbfea9

Swan, M. (2015). Blockchain: blueprint for a new economy (1st ed.). Sebastopol: O'reilly.

Szabo, N. (1997). Formalizing and securing relationships on public networks. First Monday, 2(9). Retrieved from https://ojphi.org/ojs/index.php/fm/article/ view/548/469

Teixeira, A. C. (2008). Até onde vai a participação cidadã? Le Monde Diplomatique Brasil, (7). Retrieved from https://diplomatique.org.br/ate-onde-vai-aparticipacao-cidada/

Thakur, V., Doja, M. N., Dwivedi, Y. K., Ahmad, T., \& Khadanga, G. (2019). Land records on blockchain for implementation of land titling in India. International Journal of Information Management. https://doi. org/10.1016/j.ijinfomgt.2019.04.013.

Tseng, J., Liao, Y., Chong, B., \& Liao, S. (2018) Governance on the drug supply chain via Gcoin blockchain. International Journal of Environmental Research and Public Health, 15(6), e1055. https://doi. org/10.3390/ijerph15061055.

Vieira, F. M., \& Santos, V. V. B. (2010, March). Governo eletrônico: A busca por um governo mais transparente e democrático. Congresso Consad de Gestão Pública, Brasília, DF, Brazil, 3.

Wood, G. (2014). Ethereum: a secure decentralised generalised transaction ledger. Ethereum Foundation. Retrieved from https://github.com/ethereum/wiki/wiki/ White-Paper.

Yermack, D. (2017). Corporate governance and blockchains. Review of Finance, 21(1), 7-31. https://doi. org/10.1093/rof/rfw074

Yli-Huumo, J., Ko, D., Choi, S., Park, S., \& Smolander, K. (2016). Where is current research on blockchain technology?A systematic review. PLOS ONE 11(10), e0163477. pone. 0163477

Zachariadis, M., Hileman, G., \& Scott, S. V. (2019). Governance and control in distributed ledgers: Understanding the challenges facing blockchain technology in financial services. Information and Organization, 29(2), 105-117. https://doi.org/10.1016/j. infoandorg.2019.03.001 


\section{Autores}

\section{Luzia Menegotto Frick de Moura}

Rua Washington Luiz, n 855, Centro Histórico, 90010-460, Porto Alegre, RS, Brasil.

E-mail: luziamfrick@gmail.com

(ㄴ) https://orcid.org/0000-0002-4997-7985

\section{Daniela Francisco Brauner}

Rua Washington Luiz, nº 855, Sl. 433, Centro Histórico, 90010460, Porto Alegre, RS, Brasil.

E-mail: daniela.brauner@ufrgs.br; danibrauner@gmail.com

(ㄴ) https://orcid.org/0000-0002-6379-7825

\section{Raquel Janissek-Muniz*}

Rua Washington Luiz, nº 855, Sl. 311, Centro Histórico, 90010 460, Porto Alegre, RS, Brasil.

E-mail: rjmuniz@ufrgs.br; raquel.janissek.muniz@gmail.com

(ㄴ) https://orcid.org/0000-0002-0657-6559

* Autora Correspondente

\section{Contribuições dos Autores}

$1^{\text {a }}$ autora: redatora principal do artigo. Fez o referencial teórico, a coleta, análise e sistematização dos dados, além das figuras e tabelas. Idealizadora do tema.

$2^{\mathbf{a}}$ autora: delineou o escopo inicial do estudo e método de pesquisa, auxiliando no enquadramento tecnológico das aplicações identificadas na continuidade da pesquisa. Ajudou na definição e no apoio da revisão do artigo.

$3^{\mathbf{a}}$ autora: conduziu o enquadramento do artigo para a revista, incluindo novas referências e revisando análises e discussão dos resultados. Primeira revisora, responsável pela submissão do artigo e pela correspondência com a revista.

\section{Financiamento}

Os autores informaram que não houve apoio financeiro para a pesquisa neste artigo.

\section{Conflito de Interesses}

Os autores informaram que não há conflito de interesses.

\section{Direitos Autorais}

A RAC detém os direitos autorais deste conteúdo.

\section{Verificação de Plágio}

A RAC mantém a prática de submeter todos os documentos aprovados para publicação à verificação de plágio, mediante o emprego de ferramentas específicas, e.g.: iThenticate.

\section{Método de Revisão por Pares}

Este conteúdo foi avaliado utilizando o processo de revisão por pares duplo-cego (double-blind peer-review). A divulgação das informações dos pareceristas constantes na primeira página é feita somente após a conclusão do processo avaliativo, e com o consentimento voluntário dos respectivos pareceristas. 